\title{
VENTO MASSIMO IN QUOTA SUL MEDITERRANEO
}

\author{
Felick di Benedeíl'io
}

Introduzione. - Questo lavoro ha lo scopo di fornire una teoria dei venti massimi in quota ed, in particolare, indicazioni pratiche sulle quote dei venti massimi rispetto ad alcune superficie fondamentali di riferimento (tropopausa, 500 e 300 millibar), nella zona del Mediterraneo.

Determinazione analitica della quota del vento massimo. - Nell'ipotesi che il flusso sia "geostrofico ", la grandezza $v$ della velocità del vento è espressa dalla nota formula:

$$
v=\frac{G}{l \varrho}=-\frac{1}{l \varrho} \frac{\partial p}{\partial n}
$$

dove $l=2 \omega$ sen $\phi$ è il parametro di Coriolis ( $\omega$ la velocità angolare della terra e $\varphi$ la latitudine), $\varrho$ la densità dell'aria $\mathrm{e}-\partial p / \partial n$ l'intensità del gradiente barico orizzontale.

Si osserva subito che $v$ è funzione del parametro $z$ (quota) per il tramite della densità $\varrho$, variabile con la quota ed il gradiente barico orizzontale, a sua volta funzione dell'altezza.

Allo scopo di determinare la quota alla quale $v$ diventa massimo, nell'ipotesi che le caratteristiche del moto si conservino geostrofiche a tutte le quote, occorre eguagliare a zero la derivata della [1] rispetto a $z$. Si ha, così:

$$
\frac{1}{\varrho} \frac{\partial p}{\partial n} \cdot \frac{\partial \varrho}{\partial z}-\frac{\partial}{\partial n}\left(\frac{\partial p}{\partial z}\right)=0
$$

nella quale si adottano i simboli di derivazione parziale in quanto $\varrho$ e $\partial p / \partial n$ sono variabili nei vari piani orizzontali e, quindi, funzioni non solo di $z$ ma anche delle coordinate orizzontali $x$ e $y$ e, per il teorema di Schwartz, si è invertito l'ordine di derivazione del secondo termine della [2].

Trasformiamo la [2] in modo da far comparire la variabile $T$ (temperatura assoluta) anzicchè la densità $\varrho$. Derivando logaritmicamente rispetto a $z$ l'equazione caratteristica dei gas:

$$
p=R \varrho T
$$


e, utilizzando l'equazione fondamentale della statica:

$$
\frac{1}{p} \frac{\partial p}{\partial z}-\frac{g}{R T}
$$

si ottiene l'espressione:

$$
\frac{1}{\varrho} \frac{\partial \varrho}{\partial z}=\left(\begin{array}{ll}
\gamma & g \\
R
\end{array}\right) \frac{1}{T}
$$

nella quale $\gamma=-\lambda T / \partial z$ è il gradiente termico verticale e $T$ è la temperatura assoluta.

D'altra parte:

$$
\frac{\partial}{\partial n}\left(\frac{\partial p}{\partial z}\right)=-\frac{g}{R}-\frac{\partial}{\partial n}\left(\frac{p}{T}\right)=\frac{g}{R T^{2}} p \frac{\partial \underline{T}}{\partial n}-\frac{g}{R T}-\frac{\partial_{r}^{n}}{\partial n} .
$$

Sostituendo la [5] e la [6] nella [2] e semplificando, si ottiene:

$$
\frac{1}{p} \frac{\partial p}{\partial n}-\frac{g}{R \gamma T} \frac{\partial T}{\partial n}=0
$$

che è la relazione cercata.

Supponiamo, inoltre, che, nelle condizioni più generali, la stratificazione atmosferica sia politropica, vale a dire, la temperatura $T$ sia data da:

$$
T-T_{\mathrm{o}}-\gamma z
$$

nella quale $T_{0}$ è la temperatura "al suolon e $\gamma=-\partial T / \partial z$ è il gradiente termico verticale.

Si ha:

$$
\frac{\partial T}{\partial n}=\frac{\partial T_{0}}{\partial n}-\frac{d \gamma}{d n} z
$$

D'altra parte, per un'atmosfera politropica la pressione $p$ alla quota $z$ è espressa da:

$$
p=p_{\mathrm{o}}\left(\frac{T}{T_{\mathrm{o}}}\right)^{\frac{g}{R \gamma}}
$$

Derivando logaritmicamente la [10] rispetto ad $n$, tenendo conto della [9] e semplificando, si ottiene:

$$
\frac{1}{p_{0}} \frac{\partial p_{0}}{\partial n}-\frac{g}{R \gamma} \frac{1}{T_{0}} \frac{\partial T_{0}}{\partial n}-\frac{g}{R \gamma^{2}} \frac{d \gamma}{d n} ?:: \frac{T}{T_{0}}=0
$$


Da cui:

$$
T=T_{0} e^{\left(\frac{R}{g} \gamma \frac{1}{p_{0}} \frac{\partial p_{0}}{\partial n}-\frac{1}{T_{0}} \frac{\partial T_{0}}{\partial n} ; \frac{\gamma}{d n}\right.}
$$

che, per la [8], diventa:

$$
z=\frac{T_{0}}{\gamma}\left|1-e^{\left(\frac{R}{g} v \frac{1}{p_{0}} \frac{\partial p_{0}}{\partial n}-\frac{1}{T_{0}} \frac{\partial T_{0}}{\partial n}\right) \frac{\gamma}{d \gamma} \mid}\right|
$$

Per le applicazioni, è sufficiente fermarsi al secondo termine della serie esponenziale, per cui:

$$
z=\frac{T_{0}}{\gamma}\left(\frac{1}{T_{0}} \frac{\partial T_{0}}{\partial n} \gamma--\frac{R \gamma^{2}}{g} \frac{1}{p_{0}} \frac{\partial p_{0}}{\partial n}\right) \frac{1}{\frac{d \gamma}{d n}} .
$$

Mentre l'altezza dell'atmosfera politropica (*) è espressa da $T_{\mathrm{o}} / \gamma$, l'altezza alla quale il vento geostrofico assume il massimo valore è espressa dalla [12] o dalla [13] e, quindi, com'era naturale, è una frazione dell'altezza dell'atmosfera politropica.

Dalla [13] risulta per $z$ una quantità finita anche quando $\partial T_{\mathrm{o}} / \partial n=0$ oppure $\partial p_{0} / \partial n=0$, cioè per masse d'aria orizzontalmente omogenee $o$ in assenza di vento al suolo.

L'altezza del vento geostrofico massimo risulta indeterminata nel caso particolare in cui $d \gamma / d n=0$, in quanto dalla [11] risulta:

$$
\frac{1}{p_{\mathrm{o}}} \frac{\partial p_{\mathrm{o}}}{\partial n}-\frac{g}{R \gamma} \cdot \frac{1}{T_{\mathrm{o}}} \cdot \frac{\partial T_{\mathrm{o}}}{\partial n}
$$

e, quindi, la [13] si presenterebbe nella forma indeterminata $0 / \infty$.

Condizione necessaria e sufficiente per l'esistenza del massimo. Dalla derivata prima della [1]

$$
\frac{\partial v}{\partial z}=\frac{1}{p} \frac{\partial p}{\partial n}-\frac{g}{R \gamma T} \frac{\partial T}{\partial n}
$$

(*) L'altezza dell'atmosfera politropica corrisponde alla condizione $p=0$ e si ottiene dalla [10]. Per $T_{\mathrm{o}}=273$ e $\gamma=6 \circ \mathrm{C} / \mathrm{Kn}$. $z=45.5 \mathrm{Km}$. 
si ottiene, invertendo l'ordine di derivazione:

$$
\begin{gathered}
\frac{\partial^{2} v}{\partial z^{2}}=\frac{1}{p} \frac{\partial}{\partial n}\left(\frac{\partial p}{\partial z}\right)-\frac{1}{p^{2}} \frac{\partial p}{\partial n} \frac{\partial p}{\partial z}+ \\
+\frac{g}{R \gamma} \frac{1}{T^{-}} \frac{\partial T}{\partial n} \frac{\partial T}{\partial z}-\frac{g}{R \gamma T} \frac{\partial}{\partial n}\left(\frac{\partial T}{\partial z}\right) .
\end{gathered}
$$

Per la [4] e la [6] la [14] diventa:

$$
\begin{aligned}
& \frac{\partial^{2} v}{\partial z^{2}}=\frac{g}{R T^{2}} \frac{\partial T}{\partial n}-\frac{g}{R p T} \frac{\partial p}{\partial n}+\frac{g}{R p T} \frac{\partial p}{\partial n}- \\
& -\frac{g}{R T^{2}} \frac{\partial T}{\partial n}+\frac{g}{R \gamma T} \frac{d \gamma}{d n}=\frac{g}{R \gamma T} \frac{d \gamma}{d n} .
\end{aligned}
$$

Dalla [15] risulta subito che la condizione necessaria e sufficiente per l'esistenza del vento massimo (geostrofico) in quota è che $d \gamma / d n<0$. In altre parole, la massa d'aria "fredda " deve trovarsi alla sinistra del vettore rappresentativo del vento geostrofico. Il risultato ora ottenuto concorda con le note regole relative al vento termico, nel caso particolare del vento geostrofico. Il criterio precedente è atto anche a giustificare, nel caso schematico esaminato, la esistenza della "corrente a getto $n$.

Applicazioni pratiche. - Riferiamoci al caso di una massa d'aria artica che si presenta con il gradiente termico verticale $\gamma$ eguale a $8^{\circ} \mathrm{C} / \mathrm{km}$ sull'Europa centrale e con il gradiente termico verticale eguale a $7,7^{\circ} \mathrm{C} / \mathrm{km}$ sull'Italia centrale. Ne risulta per $d \gamma / d n$, il valore medio $0,0003^{\circ} \mathrm{C} / \mathrm{km}$. Sia, inoltre:

$$
\begin{aligned}
\frac{\partial T_{0}}{\partial n} & =0,02 \mathrm{o} / \mathrm{km} \\
T_{0} & =285{ }^{\circ} \mathrm{K} \\
p_{0} & =1000 \mathrm{mb} \\
\frac{\partial p_{0}}{\partial n} & =0,035 \mathrm{mb} / \mathrm{km} .
\end{aligned}
$$

Adottando i suddetti valori nella [13], si ottiene, in conformità con le osservazioni, il seguente valore per l'altezza del vento massimo: $z=8000 \mathrm{~m}$. 
Vento massimo in quota sul mediterraneo. - E noto che in aerologia la procedura tecnica normale è aita a fornire la direzione e la velocità del vento per quote elevate soltanto in corrispondenza delle superficie isobariche principali $(500,300,200$ e $100 \mathrm{mb})$. Al contrario, la conoscenza della distribuzione dei massimi relativi dei venti in quota, limitata agli Stati Uniti, $\left({ }^{1,2}\right)$, è indispensabile per la zona del Mediterraneo, in vista del fatto che questa zona è raramente interessata dalla cosiddetta "corrente a getto ", fenomeno anemologico che ha assorbito la massima attenzione da parte dei meteorologi italiani e stranieri.

Nella scelta del materiale di spoglio, sono stati presi in considerazione 2850 sondaggi termodinamici contenenti non solo i dati relativi al vento fino a $200 \mathrm{mb}$, ma anche un tipo ben definito di tropopausa, per le seguenti stazioni del Mediterraneo:

\begin{tabular}{|c|c|c|c|c|c|}
\hline Algeri (Maison Blanche) & $36^{\circ} 43^{\prime} \mathrm{N}$ & $03^{\circ} 14^{\prime} \mathrm{E}$ & m. 28 & & \\
\hline Ankara & $399^{\circ} 57^{\prime} \mathrm{N}$ & $32^{\circ} 53^{\prime} \mathrm{E}$ & m. 891 & ” & " \\
\hline Atene (Hassani) & $37^{\circ} 54^{\prime} \mathrm{N}$ & $23^{\circ} 48^{\prime} \mathrm{E}$ & $\mathrm{m}$. & ” & \\
\hline Brindisi & $40^{\circ} 39^{\prime} \mathrm{N}$ & $17^{\circ} 57^{\prime} \mathrm{E}$ & $\mathrm{m}$. & $»$ & \\
\hline Elmas & $39015^{\prime} \mathrm{N}$ & $09^{\circ} 03^{\prime} \mathrm{E}$ & $\mathrm{m}$. & $»$ & 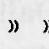 \\
\hline Malta (Qrendi) & $35^{\circ} 50^{\prime} \mathrm{N}$ & $14^{0} 27^{\prime} \mathrm{E}$ & m. 135 & $n$ & 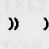 \\
\hline Messina & $38^{\circ} 12^{\prime} \mathrm{N}$ & $15^{\circ} 33^{\prime} \mathrm{E}$ & $\mathrm{m}$. & $"$ & \\
\hline Milano (Linate) & $45^{\circ} 28^{\prime} \mathrm{N}$ & $09^{\circ} 17^{\prime} \mathrm{E}$ & m. 121 & $"$ & 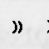 \\
\hline Nimes & $43^{\circ} 51^{\prime} \mathrm{N}$ & $04^{\circ} 24^{\prime} \mathrm{E}$ & $\mathrm{m}$. & 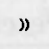 & 1 \\
\hline Roma (Ciampino) & $41^{\circ} 48^{\prime} \mathrm{N}$ & $12^{\circ} 35^{\prime} \mathrm{E}$ & m. 122 & $"$ & $"$ \\
\hline
\end{tabular}

Sulla scorta di questi dati, sono stati tracciati alcuni diagrammi che verranno illustrati in seguito.

Per poter fornire indicazioni precise circa l'interpretazione dei diagrammi riportati, occorre tener presente quanto segue.

La tropopausa, definita una volta come la superficie di separazione fra la troposfera e la stratosfera, si presenta, da ricerche dettagliate recenti (Byers $\left({ }^{3}\right)$, Flohn e Penndorf $\left({ }^{4}\right)$ ) non come una superficie, ma piuttosto come uno strato d'aria. In questo strato, poichè il gradiente termico verticale $\gamma$ può essere $\leqslant 0$, la tropopausa assume rispettivamente una delle seguenti caratteristiche:

$\begin{array}{lll}\text { Tipo I } & \text { (normale) } & \partial T / \partial z=0 \\ \text { Tipo II } & \text { (ascendente) } \partial T / \partial z>0 \\ \text { Tipo III (subsidente) } & \partial T / \partial z<0\end{array}$


In verità, non sempre in pratica l'esame della curva di stato conduce categoricamente ad uno dei tre tipi precedenti. Poichè l'individuazione della tropopausa "principale» e, quindi, della sua altezza, risulta problematica, sono stati scartati tali casi dalla serie dei 2850 sondaggi scelti.

$\mathrm{Nel}$ complesso, la tropopausa viene individuata in base alla seguente definizione $\left({ }^{1}\right)$ :

"La tropopausa è situata nel punto più basso del sondaggio, dove il gradiente termico verticale decresce fino a $2^{\circ} \mathrm{C} / \mathrm{km}$ d'altezza o meno e presenta un valore medio minore o eguale a $2^{\circ} \mathrm{C} / \mathrm{km}$ per i primi due $\mathrm{km}$ d'altezza al di sopra del suddetto punto ".

Fanno eccezione gli strati medi e bassi stabili della troposfera e degli strati frontali, facilmente riconoscibili dalla carta generale del tempo.

Deserizione dei diagrammi. - Nella fig. 1, si riportano in ascisse le differenze $H_{T}-H_{M}$ in $\mathrm{km}$, fra l'altezza della tropopausa e l'altezza del vento massimo, per le stazioni di radiosondaggio suddetto, per tutte le stagioni e a prescindere dai tipi di propopausa: in ordinate le frequenze percentuali delle differenze riportate in ascisse.

Dalla fig. 1 risulta un massimo assoluto $(33,4 \%)$ in corrispondenza della tropopausa e di altri due massimi (dell'ordine del $5 \%$ ) a due-tre $\mathrm{km}$ al di sopra e al di sotto della tropopausa. Esiste, inoltre, una serie di massimi relativi $(1-2 \%)$ la cui presenza si spiega per il fatto che sono stati presi in considerazione tutti $i$ valori relativi alla velocità del vento senza prescindere dalla località o dal tipo di tropopausa.

Nella fig. 2, la distribuzione percentuale è relativa alla differenza $H_{M}-H_{500}$ (differenza fra l'altezza del vento massimo e altezza della superficie di $500 \mathrm{mb}$, mentre nella fig. 3 il diagramma è relativo alla differenza $H_{M}-H_{300}$ (differenza fra l'altezza del vento massimo e l'altezza della superficie di $300 \mathrm{mb}$ per le stesse stazioni di radiosondaggio della fig. 1 e per tutte le stagioni.

Questi diagrammi relativi alle figg. 2 e 3 sono di notevole importanza in quanto forniscono la posizione del vento massimo rispetto alle superficie di riferimento con procedimento scevro d'incertezze, come accade, riferendo il vento massimo alla tropopausa. Essi danno, inoltre, precise indicazioni circa l'utilità di queste superficie che sono alla base dei servizi aerologici nazionali ed internazionali, agli effetti dell'indicazione della quota "economica» di volo. La fig. 2 mostra chiaramente l'esistenza di due massimi $(6,7 \%)$ al di sopra della superficie di $500 \mathrm{mb}$ e un altro massimo $(3 \%)$ in corrispondenza della superficie stessa. E evidente l'esistenza dei massimi al di sopra della superficie di $500 \mathrm{mb}$, 


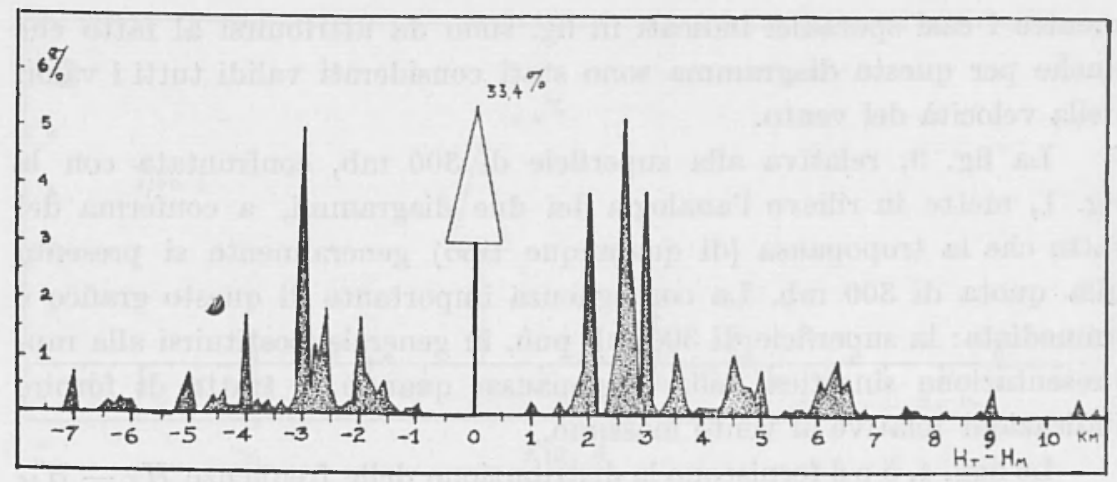

Fig. 1

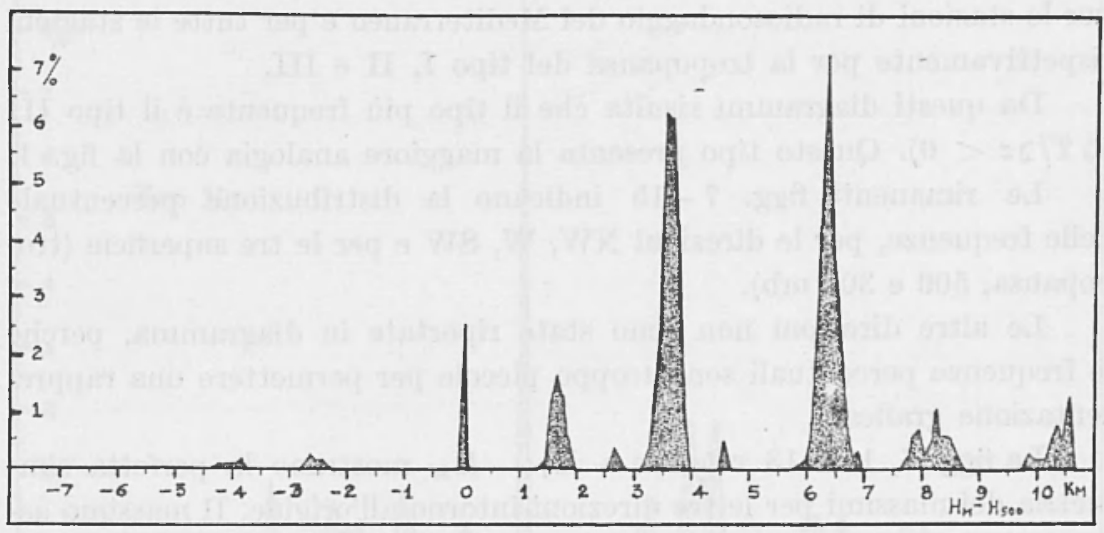

Fig. 2

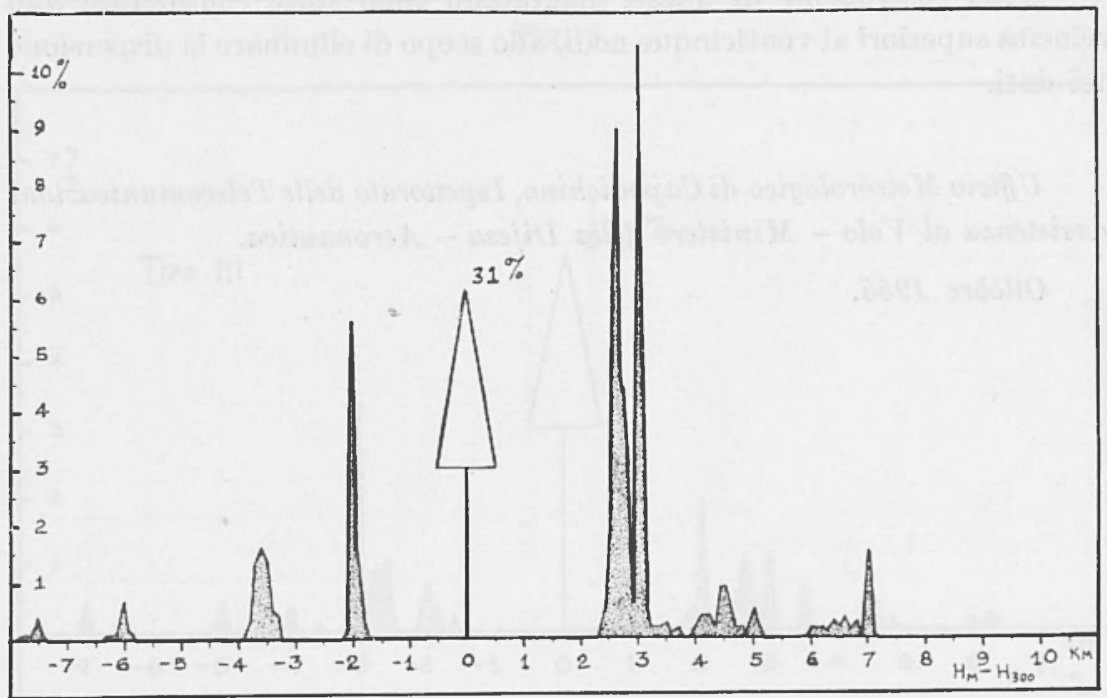

Fig. 3 
mentre i casi sporadici indicati in fig. sono da attribuirsi al fatto che anche per questo diagramma sono stati considerati validi tutti $i$ valori della velocità del vento.

La fig. 3, relativa alla superficie di $300 \mathrm{mb}$, confrontata con la fig. 1 , mette in rilievo l'analogia dei due diagrammi, a conferma del fatto che la tropopausa (di qualunque tipo) generalmente si presenta alla quota di $300 \mathrm{mb}$. La conseguenza importante di questo grafico è immediata: la superficie di $300 \mathrm{mb}$ puó, in generale, sostituirsi alla rappresentazione sinottica della tropopausa, quando si tratta di fornire indicazioni relative al vento massimo.

Le figg. 4, 5 e 6 forniscono la distribuzione delle frequenze $H_{T}-H_{M}$ (differenza fra l'altezza della tropopausa e l'altezza del vento massimo) per le stazioni di radiosondaggio del Mediterraneo e per tutte le stagioni rispettivamente per la tropopausa del tipo I, II e III.

Da questi diagrammi risulta che il tipo più frequente è il tipo III ( $T / \partial z<0$ ). Questo tipo presenta la maggiore analogia con la fig. 1 .

Le rimanenti figg. $7-15$ indicano la distribuzione percentuale delle frequenze, per le direzioni $\mathrm{NW}, \mathrm{W}, \mathrm{SW}$ e per le tre superficie (tropopausa, 500 e $300 \mathrm{mb}$ ).

Le altre direzioni non sono state riportate in diagramma, perchè le frequenze percentuali sono troppo piccole per permettere una rappresentazione grafica.

Le figg. 7,10 e 13 relative a $H_{T}-H_{M}$ mostrano la perfetta simmetria dei massimi per le tre direzioni intorno all'origine. Il massimo assoluto $(10,3 \%)$ è relativo al vento occidentale.

Nella costruzione di questi diagrammi sono state considerate solo velocità superiori ai venticinque nodi, allo scopo di eliminare la dispersione dei dati.

Ufficio Mcteorologico di Capodichino, Ispettorato delle Telecomunicazioni Assistenza al Volo - Ministero della Difesa - Aeronautica.

Ottobre 19.5 .5 . 
VENTo MASSIMO IN QUOTA SUL MEDITERRANEO

387

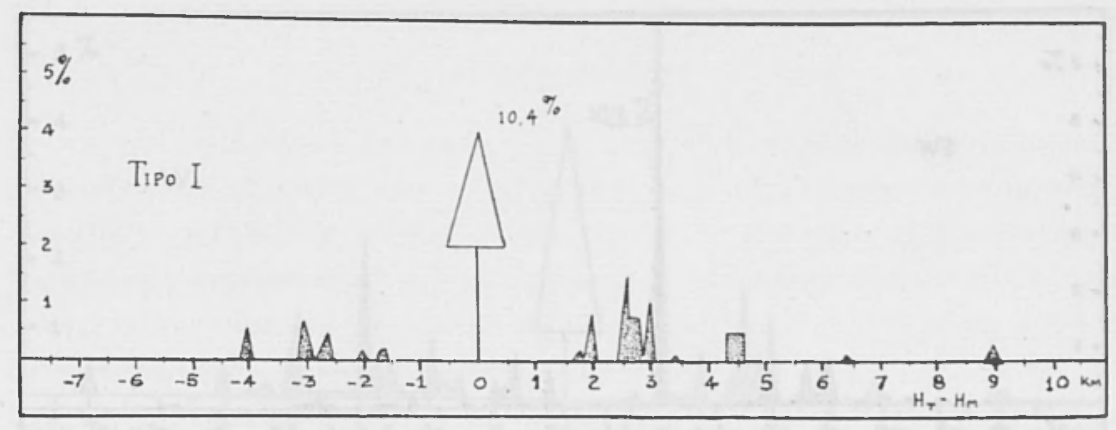

Fig. 4

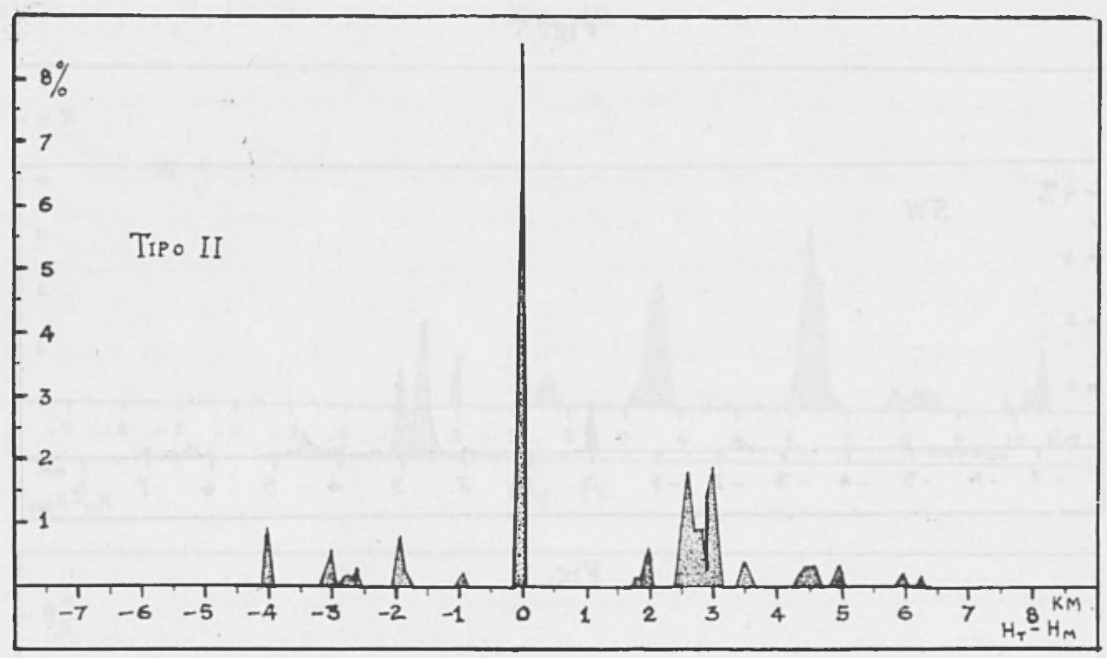

Fig. 5

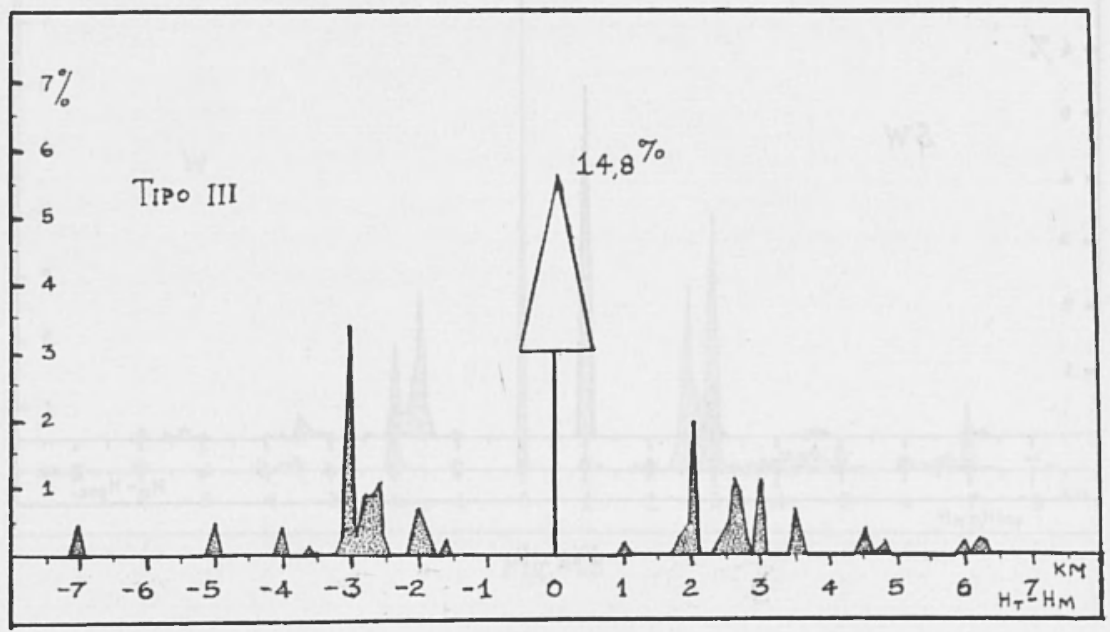

Fig. 6 


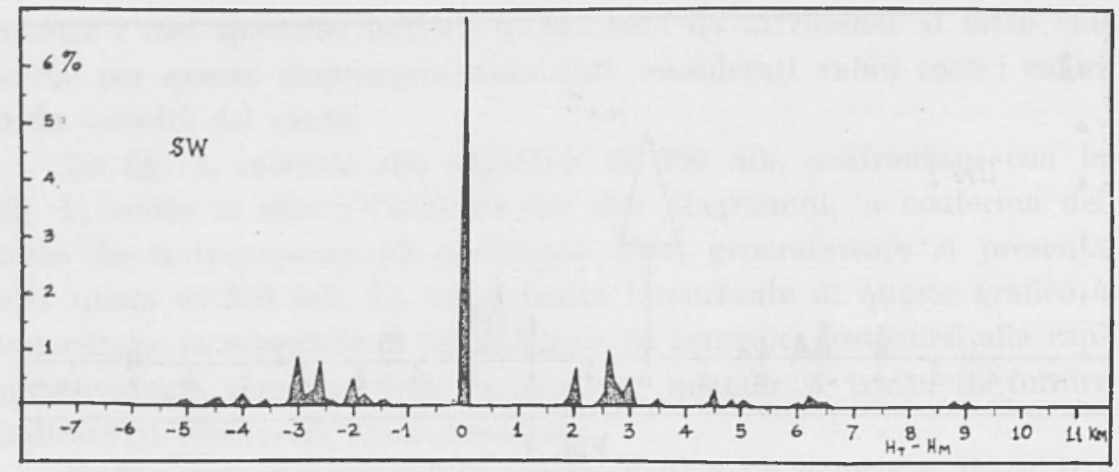

Fig. 7

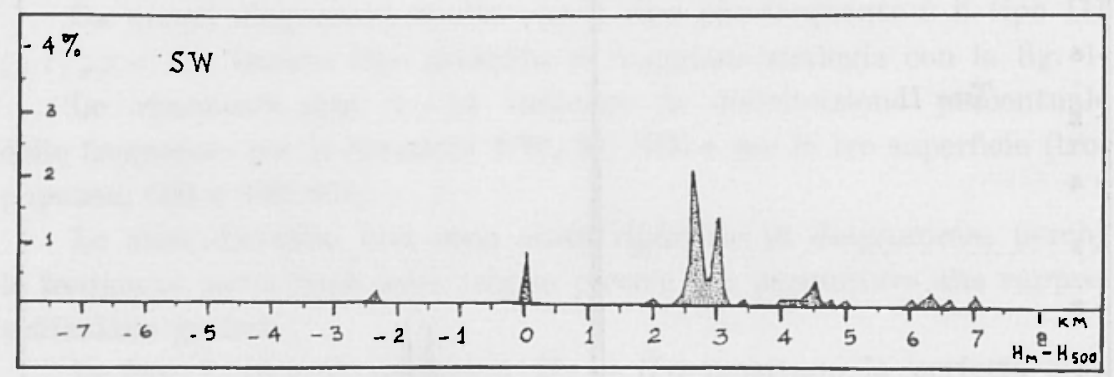

Fig. 8

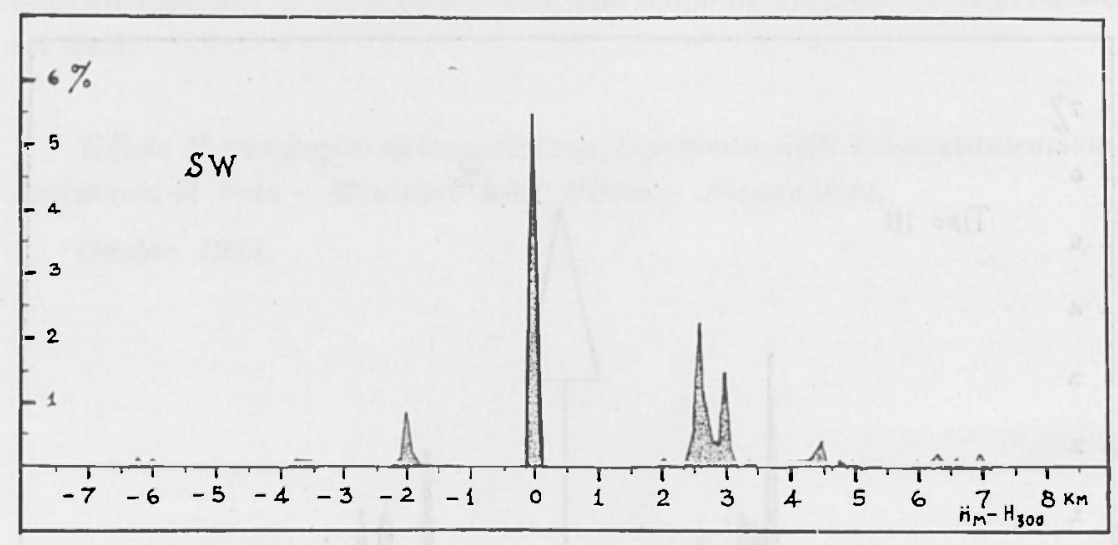

Fig. 9 


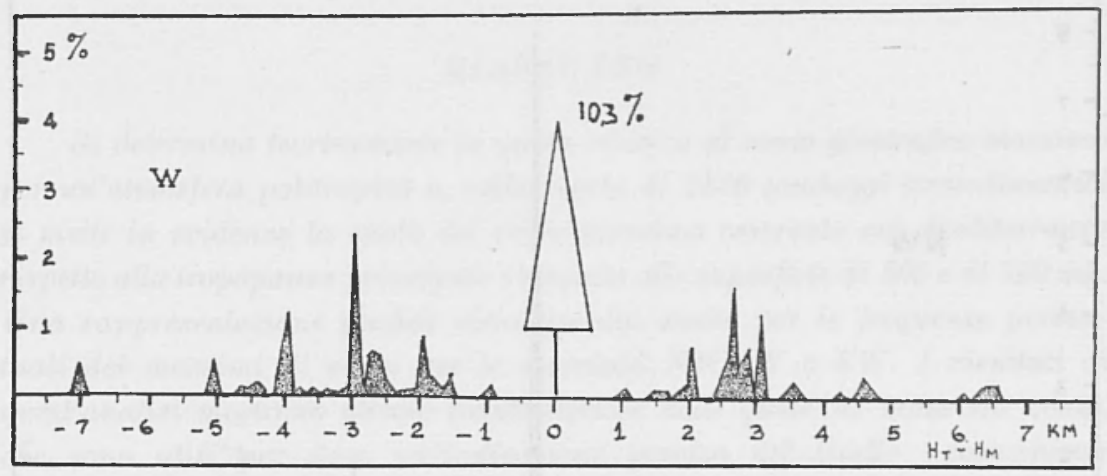

Fig. 10

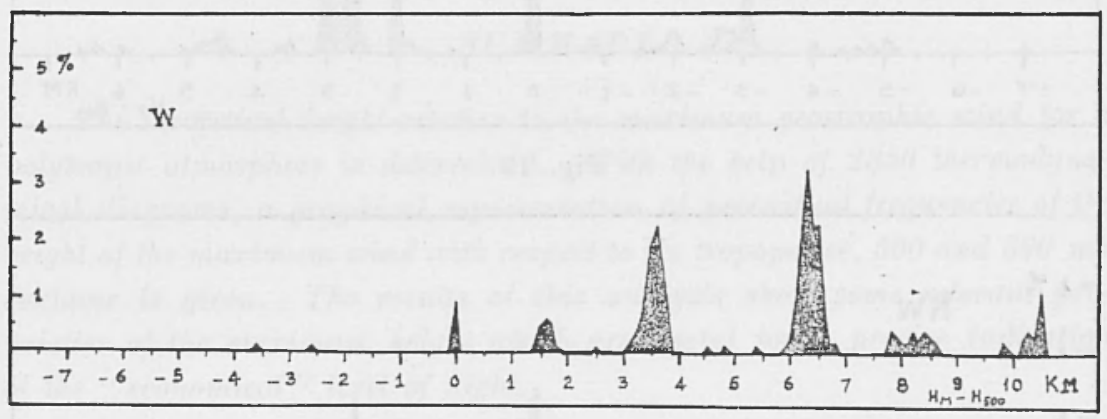

Fig. 11

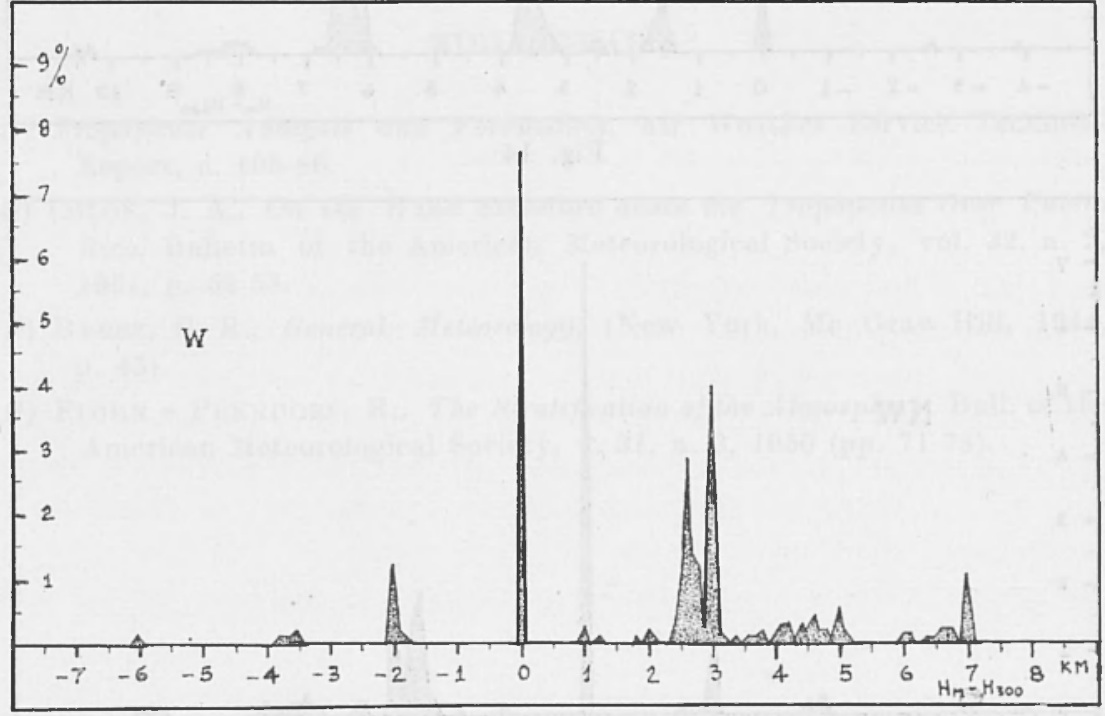

Fig. 12 


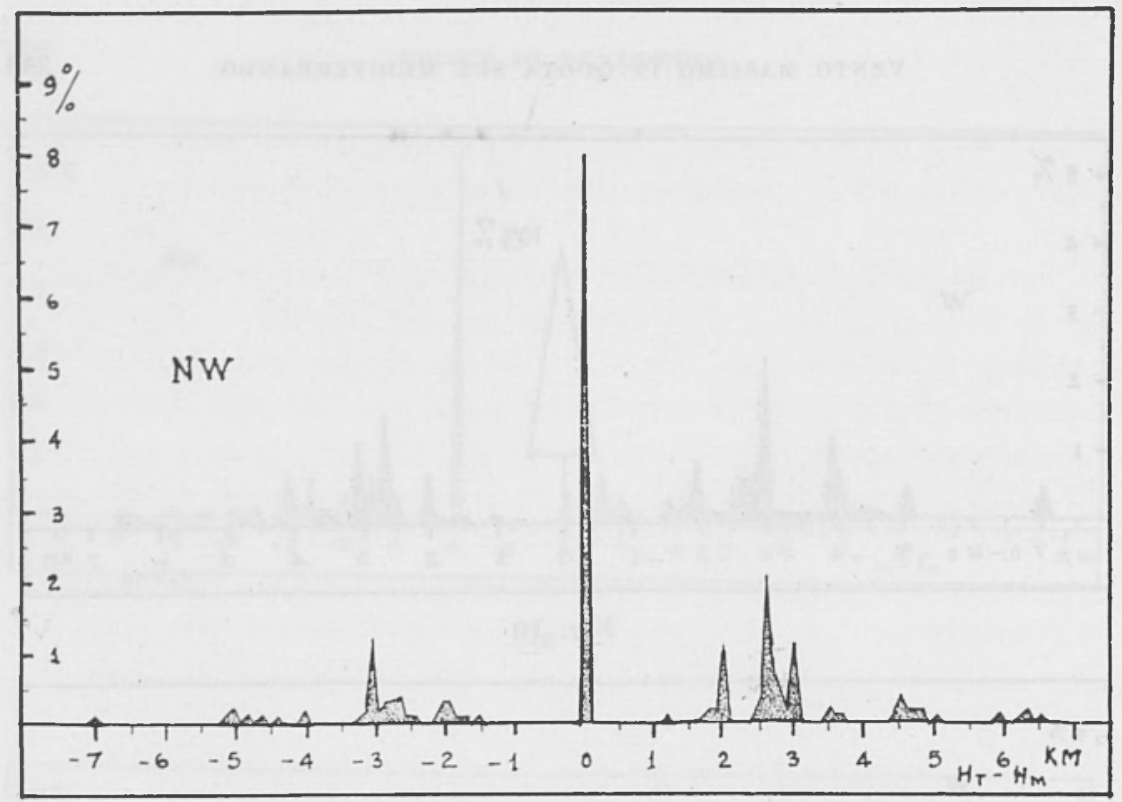

Fig. 13

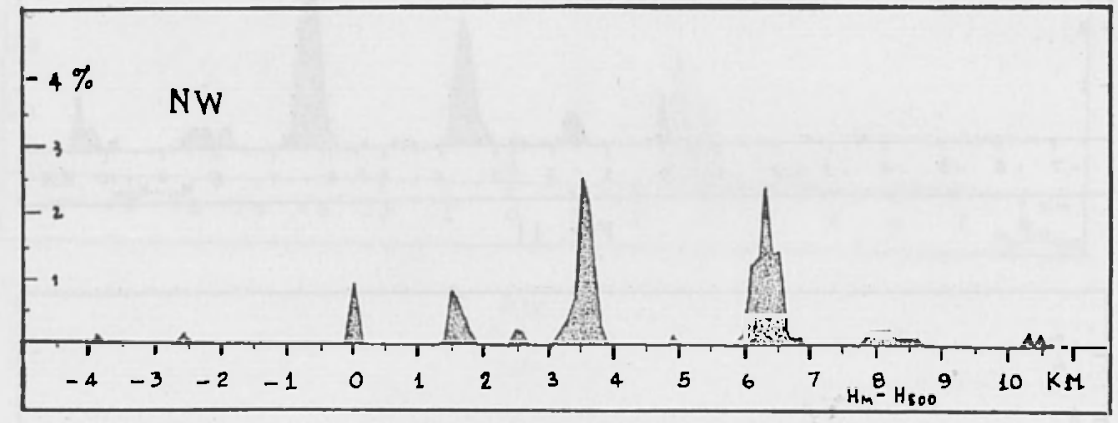

Fig. 14

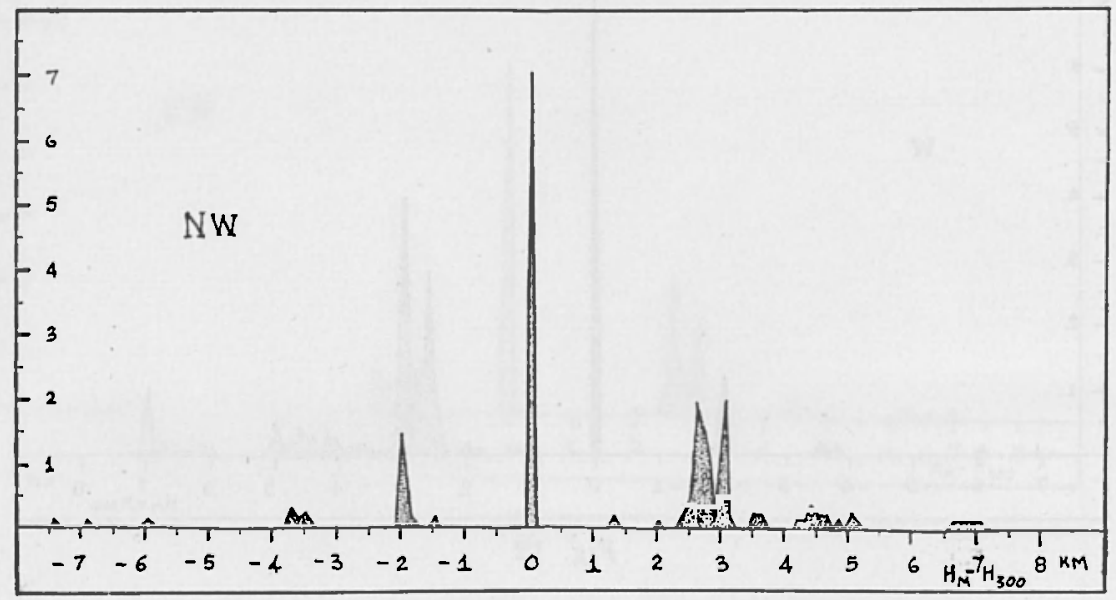

Fig. 15 


\section{RIASSENTO}

Si determina teoricamente la quota relativa al vento geostrofico massimo per un'atmosfera politropica e, sulla scorta di 2850 sondaggi termodinamici si mette in evidenza la quota del vento massimo osservato sul Mediterraneo rispetto alla tropopausa principale e rispetto alle superficie di 500 e di $300 \mathrm{mb}$. Una rappresentazione grafica viene fornita anche per le frequenze percentuali dei massimi di vento per le direzioni $N \mathrm{~W}$, W e SW. I risultati di quest'analisi mostrono alcune caratteristiche delle quote di massimo vento, che sono utili per dare un'indicazione precisa del livello "economico" di volo.

\section{$S E M M A R I$}

The theoretical height relative to the maximum geostrophic wind for a polytropic atmosphere is determined. With the help of 2850 thermodynamical diagrams, a graphical representation of percentual frequencies of the height of the maximum wind with respect to the tropopause, j00 and $300 \mathrm{mb}$ surfaces is given. The results of this analysis show some relevant proprieties of the maximum height which are useful for a precise indication of the "economical" level of flight.

\section{BIBLIOGRAFIA}

(1) Tropopause Analysis and Forecasting, Air Weather Service Technical Report, n. 105.86.

(2) Colon, J. A., On the IIind Structure above the Tropopause Over Puerto Rico, Bulletin of the American Meteorological Society, vol. 32, n. 2, 1951 , p. $52-53$.

( ${ }^{3}$ ) Byers, H. R., General Meteorology, (New York, Mc Graw-Hill, 1944, p. 45$)$.

(4) FLOHx e PENNDORJ, R., The Stratification of the dimosphere. Bull. of the American Meteorological Society, v. 31. n. 3, 1950 (pp. 71-78). 